\title{
Simulated Disperser Analysis: Determining the number of loci required to genetically identify dispersers
}

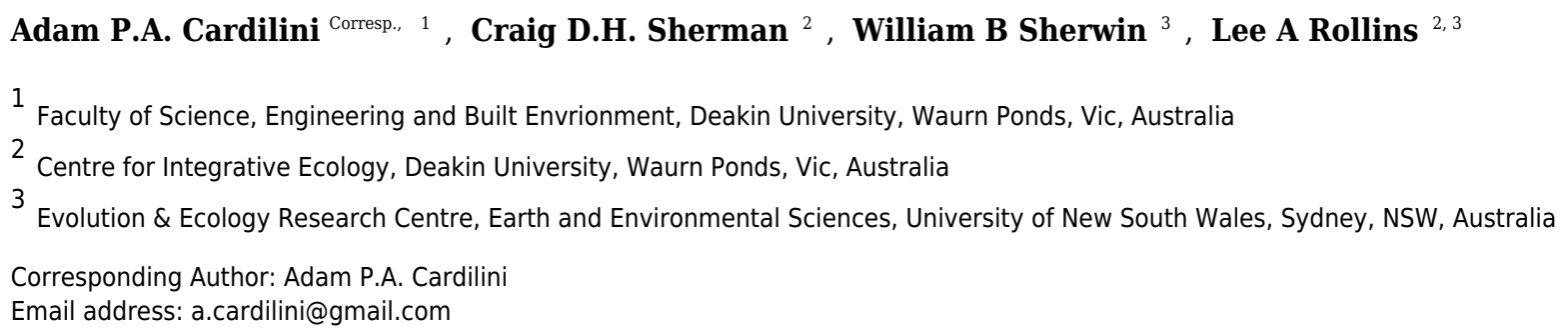

Empirical genetic datasets used for estimating contemporary dispersal in wild populations and to correctly identify dispersers are rarely tested to determine if they are capable of providing accurate results. Here we test whether a genetic dataset provides sufficient information to accurately identify first-generation dispersers. Using microsatellite data from three wild populations of common starlings (Sturnus vulgaris), we artificially simulated dispersal of a subset of individuals; we term this 'Simulated Disperser Analysis'. We then ran analyses for diminishing numbers of loci, to assess at which point simulated dispersers could no longer be correctly identified. Not surprisingly, the correct identification of dispersers varied significantly depending on the individual chosen to 'disperse', the number of loci used, whether loci had high or low Polymorphic Information Content and the location to which the dispersers were moved. A review of the literature revealed that studies that have implemented first-generation migrant detection to date have used on average 10 microsatellite loci. Our results suggest at least 27 loci are required to accurately identify dispersers in the study system evaluated here. We suggest that future studies use the approach we describe to determine the appropriate number of markers needed to accurately identify dispersers in their study system; the unique nature of natural systems means that the number of markers required for each study system will vary. Future studies can use Simulated Disperser Analysis on pilot data to test marker panels for robustness to contemporary dispersal identification, providing a powerful tool in the efficient and accurate design of studies using genetic data to estimate dispersal. 


\section{Simulated Disperser Analysis: determining the number of loci required to}

\section{2 genetically identify dispersers.}

3 Adam P.A. Cardilini' ${ }^{1}$, Craig D.H. Sherman², William B. Sherwin ${ }^{3}$, Lee A. Rollins ${ }^{2,3}$

$4 \quad{ }^{1}$ Faculty of Science, Engineering and Built Environment, Deakin University, 75 Pigdons Rd,

5 Waurn Ponds, Victoria 3216, Australia

$6{ }^{2}$ Centre for Integrative Ecology, School of Life and Environmental Science, Deakin University,

775 Pigdons Rd, Waurn Ponds, Victoria 3216, Australia

$8 \quad{ }^{3}$ Evolution \& Ecology Research Centre, Earth and Environmental Sciences, The University of

9 New South Wales, Sydney, NSW 2052, Australia

11 Corresponding Author:

12 Adam P.A. Cardilini

13 Faculty of Science, Engineering and Built Environment, Deakin University, 221 Burwood Hwy,

14 Burwood, Victoria 3125, Australia

15 Email: a.cardilini@gmail.com

16 


\section{Abstract}

18 Empirical genetic datasets used for estimating contemporary dispersal in wild populations and to

19 correctly identify dispersers are rarely tested to determine if they are capable of providing

20 accurate results. Here we test whether a genetic dataset provides sufficient information to

21 accurately identify first-generation dispersers. Using microsatellite data from three wild

22 populations of common starlings (Sturnus vulgaris), we artificially simulated dispersal of a

23 subset of individuals; we term this 'Simulated Disperser Analysis'. We then ran analyses for

24 diminishing numbers of loci, to assess at which point simulated dispersers could no longer be

25 correctly identified. Not surprisingly, the correct identification of dispersers varied significantly

26 depending on the individual chosen to 'disperse', the number of loci used, whether loci had high

27 or low Polymorphic Information Content and the location to which the dispersers were moved. A

28 review of the literature revealed that studies that have implemented first-generation migrant

29 detection to date have used on average 10 microsatellite loci. Our results suggest at least 27 loci

30 are required to accurately identify dispersers in the study system evaluated here. We suggest that

31 future studies use the approach we describe to determine the appropriate number of markers

32 needed to accurately identify dispersers in their study system; the unique nature of natural

33 systems means that the number of markers required for each study system will vary. Future

34 studies can use Simulated Disperser Analysis on pilot data to test marker panels for robustness to

35 contemporary dispersal identification, providing a powerful tool in the efficient and accurate

36 design of studies using genetic data to estimate dispersal. 


\section{Introduction}

39 Dispersal is a major contributor to population processes, and can strongly influence genetic

40 diversity, local adaptation, speciation, levels of inbreeding, sexual selection and many other

41 biologically important processes (Clobert et al. 2012). Knowledge of dispersal patterns can

42 improve evaluation of the maintenance of source-sink population dynamics (Walsh et al. 2012),

43 the identification of habitat corridors or barriers to movement (Dzialak et al. 2005; Gortat et al.

44 2009), the determination of biological limitations to dispersal (e.g. sex biased dispersal, Goudet

45 et al. 2002), and our understanding of how the environment affects dispersal behaviour (Pruett-

46 Jones \& Lewis 1990). Such knowledge can contribute to a theoretical understanding of

47 ecological processes and be applied to improve conservation management practices (Fahrig \&

48 Merriam 1994; Selonen et al. 2010; Keller et al. 2010). For over a century, direct measures of

49 dispersal have been collected using mark-recapture data (Sandercock 2003), and more recently

50 using telemetry techniques (Harris et al. 1990) and Geographic Position Systems (GPS) tracking

51 (Cagnacci et al. 2010). Unfortunately, these methods have practical constraints, including

52 expense and the difficulty of deploying and retrieving a large number of units. Alternatively,

53 genetic methods that identify individual contemporary dispersers are commonly used because

54 they are relatively cheap and easy to implement (Broquet \& Petit 2009). These methods include

55 Bayesian statistics to identify the probability that a particular genotype arises from each of a

56 group of populations (Pritchard et al. 2000; Piry et al. 2004; Jombart et al. 2008). If an

57 individual's genotype has alleles that are more common to a population other than where it was

58 sampled, the individual is considered to be a potential disperser (Rannala \& Mountain 1997).

59 The accuracy of genetic assignment methods depends on the demographic parameters of the

60 species being investigated (e.g. rates of dispersal; Bohonak 1999; Whitlock \& McCauley 1999), 
61 experimental design (e.g. proportion of populations sampled, number of individuals sampled;

62 Cornuet et al. 1999; Paetkau et al. 2004; Schwartz \& McKelvey 2008), and the markers chosen

63 (e.g. levels of polymorphism, number of loci; Berry et al 2004). Given the number of factors that

64 can influence the correct identification of dispersers using genetic assignment techniques, it is

65 important that studies robustly test if the marker system and population structure allow for

66 reliable identification and assignment of dispersers to the populations being studied. For

67 example, Berry et al. (2004) were able to determine the number of loci needed to accurately

68 assign dispersers of grand skink (Oligosoma grande) populations by directly comparing genetically

69 identified dispersers with mark-recapture data collected over a seven year period. Ideally,

70 estimates of dispersal between populations should be carried out using multiple approaches to

71 confirm congruence between estimation methods; however, most ecological studies are restricted

72 to using a single approach either due to logistical or budget constraints (or both). Genetic data

73 may permit the identification of dispersers and assignment to their source populations, but also

74 provide the ability to estimate a range of other population parameters often of interest to

75 ecological researchers (e.g. estimates of genetic diversity and heterozygosity, levels of

76 inbreeding, multiple paternity, and historical patterns of connectivity; Excoffier et al. 2005;

77 Peakall \& Smouse 2006; Porras-Hurtado et al. 2013; Waser \& Hadfield 2011; Waser et al.

78 2001).

79 Here we perform a Simulated Disperser Analysis, using microsatellite data from three Australian

80 common starling (Sturnus vulgaris) populations to test the power of a marker system to

81 accurately identify 'dispersers' (individuals that are not native to their collection locality) using

82 genetic assignment tests and genetic data only. This approach simulates dispersal between

83 populations and then tests a number of parameters (number of loci, levels of variability of loci, 
84 and effect of disperser genotype) on the ability to identify dispersers and to correctly assign these

85 to their collection locality.

\section{Methods}

\section{Ethics statement}

88 The collection of all samples followed the strict guidelines outlined in the ethics application

89 (permit number: 05/011A) approved by The University of New South Wales ethics committee.

\section{Sample collection and DNA methods}

91 We investigated dispersal in Australian invasive starlings (Sturnus vulgaris), a highly vagile

92 species that maintains an extensive distribution across southeast Australia. Samples were taken

93 from three populations previously shown to have low levels of genetic differentiation (Rollins et

94 al. 2009; $F_{S T}=0.02$ - 0.07): Orange (New South Wales, 33 17' $\mathrm{S}, 1^{\circ} 4^{\circ} 06^{\prime} \mathrm{E}, N=32$ ); Mallala

95 (South Australia, 34 27' S, 138 30' E, $N=32$ ); and Munglinup (Western Australia, 33' 42' 29'”

$\left.96 \mathrm{~S}, 120^{\circ} 51^{\prime} 54^{\prime \prime} \mathrm{E}, N=30\right)$.

DNA was extracted using Gentra PureGene DNA extraction kit (Qiagen) following the manufacturer's instructions. Microsatellites were developed using next generation sequencing on the GS-FLX 454 platform (Roche, Manheim, Germany) following methods described by

100 Abdelkrim et al. (2009). QDD v 0.9.0.0 Beta (Meglécz et al. 2010) was used to identify

101 microsatellites from the sequencing data and primers were designed using the program PRIMER

1023 (Rozen \& Skaletsky 2000). A panel of 20 polymorphic markers was chosen (see

103 Supplementary Table S1). PCR reactions were multiplexed using universal fluorescently labelled

104 primers (Neilan et al. 1997). A step-down PCR protocol was used for each reaction consisting of 105 ten cycles each at the following annealing temperatures: $70^{\circ} \mathrm{C}, 64{ }^{\circ} \mathrm{C}, 58^{\circ} \mathrm{C}, 54^{\circ} \mathrm{C}, 50^{\circ} \mathrm{C}$. Reactions 
106 containing DNA from the same individual but with differently labelled universal markers (e.g.

107 PET, NED, 6-FAM and VIC) were combined in equivalent amounts, so that all loci for each

108 individual could be multi-loaded into three tubes prior to fragment analysis (see Supplementary

109 Table S1). Samples were genotyped using an ABI 3730 (Applied Biosystems, Foster City, CA,

110 USA) using GS-500 (Liz) in each capillary as a size standard. Allele sizes were estimated on

111 GENEMAPPER version 3.7 (Applied Biosystems). These data were combined with data from 11

112 microsatellite loci for the same individuals as detailed in Rollins et al. (2009) giving us a total of

11331 loci.

114 Microsatellite markers were checked for departures from Hardy-Weinberg equilibrium (Arlequin

115 version 3.5.1.2, Excoffier et al. 2005) and linkage disequilibrium (GenePop version 4.0.10,

116 Rousset 2008). PIC was calculated for each locus using PICcalc (Nagy et al. 2012). Arlequin

117 was used to calculate Pairwise $F_{S T}$ values. Pairwise values for Shannon's mutual information

118 index (I, formerly called ${ }^{S} H_{U A}$, Sherwin et al. 2017) were calculated in GenAlEx (Peakall \&

119 Smouse 2006) because mutual information is better than $F_{S T}$ at handling a range of population

120 sizes and dispersal rates (Sherwin et al. 2006; Dewar et al. 2011).

121 Ninety-four samples were successfully genotyped for 31 microsatellite loci. Of the 20 new

122 species-specific loci developed here, two showed significant deviation from Hardy-Weinberg

123 equilibrium (Svu002 and Svu010) and were removed from further analyses (see Supplementary

124 Table 3). None of the remaining 18 loci showed significant departures from linkage equilibrium

125 after Bonferroni correction. Data from the other 11 loci previously have been shown to be in

126 Hardy-Weinberg equilibrium in these populations by Rollins et al. (2009). 


\section{Simulations}

128 Initially, we used GeneClass2 (Piry et al. 2004) to determine the probability of each individual

129 originating in the population where it was sampled (see method below). One individual from

130 each population was randomly assigned to be a 'simulated disperser'; individuals with low

131 probabilities of population membership were excluded from being simulated dispersers because

132 they were potentially real dispersers. Simulated dispersers were moved in every possible

133 combination relative to other simulated dispersers creating a total of $27\left(3^{3}\right)$ treatments

134 (including a treatment of no movement, Table 1).

135 Loci were ranked by their Polymorphic Information Content (PIC) (see statistical analysis

136 below). Each treatment was analysed using 57 genetic datasets. The first analysis started with the

137 locus with the highest PIC and then consecutively adding the next highest PIC locus until all loci

138 were added ( $N=29$, including the dataset having all markers; referred to as highest PIC hereafter).

139 The second analysis started with the locus with the lowest PIC and then consecutively adding the

140 next lowest PIC locus until all loci were added $(N=28$, because the dataset with all markers was

141 analysed in the previous analysis; referred to as lowest PIC hereafter).

142 The partial Bayesian method (Rannala \& Mountain 1997) implemented in GeneClass2.0 was

143 used to detect simulated dispersers. We ran 10,000 MCMC simulations per population using the

$144 L_{h} / L_{\max }$ likelihood computation (Paetkau et al. 2004). Individuals were considered first-

145 generation dispersers if they had an $L_{h} / L_{\max } p$-value that was below 0.01 . An individual can

146 incorrectly be identified as a disperser in two ways: if they are identified as a disperser when

147 they are not one (False Positive), or, when they fail to be identified as a disperser when they are

148 one (False Negative). Once identified, GeneClass2.0 assigns dispersers to the most likely source 149 population. 
150 In our assessment of the ability of GeneClass 2.0 to detect simulated migrants, assignment tests

151 were repeated for treatments and individuals, resulting in the non-independence of data. To

152 account for this, we used Generalised Linear Mixed Models (GLMMs), specifying treatment and

153 individual as random effects, and predictor variables (number of loci, highest / lowest PIC loci

154 used, location the simulated disperser was moved to, and I) as fixed effects (Zuur et al. 2009).

155 The response variable 'dispersal status correctly identified' was a binary indicator of the ability

156 of GeneClass 2.0 to correctly identify whether a simulated disperser was a disperser or not $(0=$

157 incorrectly identified, 1 = correctly identified) and was specified as having a binomial

158 distribution of errors with a logit link function. For example, if an individual was identified as a

159 resident in the population from which it was sampled, it was classed as 'dispersal status correctly

160 identified'. The response variable (dispersal status correctly identified) was modelled as a

161 function of a) the number of loci, b) whether the highest / lowest PIC loci were used, c) the

162 population in which the simulated disperser was located (either its collection locality or one of

163 the other two populations) and d) the pairwise I value between populations when moved. The

164 number of loci was a discrete numeric variable, while highest / lowest PIC loci used (PIC range

$165=0.251-0.862$ ) was specified as a categorical variable with two levels, location was specified as

166 a categorical variable where individuals were coded as either being from their collection locality

167 or one of the other two populations (collection locality was used as the reference group with

168 which other groups were compared), and pairwise I was specified as a continuous numeric

169 variable. Model fit was estimated from marginal $\left(\mathrm{R}^{2}{ }_{\mathrm{GLMM} /(\mathrm{m})}\right)$ and conditional $\left(\mathrm{R}^{2} \mathrm{GLMM} /(\mathrm{c})\right)$

170 coefficients of determination, following Nakagawa and Schielzeth (2013). $\mathrm{R}_{\mathrm{GLMM} /(\mathrm{m})}$ estimates

171 model fit using fixed effects only, while $\mathrm{R}^{2}{ }_{\mathrm{GLMM} /(\mathrm{c})}$ estimates model fit including both fixed and 
172 random effects. By comparing these estimates it is possible to compare the contribution that

173 random effects and fixed effects have on the response variable.

\section{Literature search}

175 To assess how studies have addressed parameters that affect power to detect dispersers (number

176 of individuals, number of loci, levels of genetic differentiation) we used Google Scholar to

177 conduct a literature search (on 19/12/2016) for articles that cited the publication that originally

178 presented the GeneClass 2 analysis (Piry et al. 2004). We used the key term 'dispersal' to narrow

179 the search and of 1,280 articles identified, we randomly chose 160 articles to assess. If the article

180 did not use GeneClass2.0's migrant detection analysis we disregarded it; if the article did use this

181 method we collected information on the number of loci used, global $F_{S T}$ value, and general

182 article meta-data such as author(s), year published, common name and species name. Of the 160

183 papers that were assessed, 132 matched the selection criteria, including a total of 136 datasets

184 (Supplementary Table S2).

185 Generalised Linear Models (GLMs) were used to determine the relationship between the global

$186 F_{S T}$ values of studies using genetic assignment tests and the number of loci that were used by

187 these studies to identified dispersers. The number of loci used was specified as a continuous

188 response variable, with a Poisson distribution, which was determined graphically and the global

$189 F_{S T}$ value was a fixed effect.

190 To demonstrate the continuing relevancy of this work (see De Barba et al 2016), we searched

191 within the 1,280 articles we identified above for those containing the search term 'microsatellite'

192 by year since this technique was published (2005-2016) to determine whether the number of

193 papers using this approach was decreasing over time. 


\section{Statistical analysis}

195 Regression models and graphics were generated using the statistical programming environment

$196 \mathrm{R}$ (version 2.15.2, R Development Core Team 2012). GLMMs were generated using the function

197 glmer within the package lme4 (version 0.999999-2, Bates et al. 2012). Graphics were generated

198 using the package ggplot2 (version 0.9.3.1, Wickham 2009).

\section{Results}

200 Sample inclusion and genetic analysis

201 Populations from Orange (New South Wales) and Mallala (South Australia) showed the lowest

202 levels of differentiation from each other $\left(F_{S T}=0.026, P\right.$ value $\left.<0.001 ; \mathrm{I}=0.081\right)$, while the

203 population from Munglinup (Western Australia) displayed higher levels of genetic differentiation

204 from Orange $\left(F_{S T}=0.082, P\right.$ value $\left.=<0.001 ; \mathrm{I}=0.152\right)$ and Mallala $\left(F_{S T}=0.081, P\right.$ value $=$

$205<0.001 ; \mathrm{I}=0.139)$ indicating that Munglinup is the most genetically distinct area sampled.

206 One of 94 individuals was identified as a potential disperser within the original dataset, which

207 included all loci and no simulated dispersal. We avoided using this individual as a simulated

208 disperser.

\section{Simulations}

210 The simulation results showed that the ability to correctly identify a simulated disperser was

211 strongly associated with an increase in the number of loci used and when the highest PIC loci

212 were used rather than the lowest (Table 2, Fig. 1). For a subset of the simulation data (treatments

213 where simulated dispersers were moved outside their collection locality), I values showed a

214 strong positive relationship with correct identification of simulated dispersers. When a simulated

215 disperser was moved to a location that had a relatively high pairwise I to its collection locality, it 
216 was more likely to be correctly identified as a disperser (Table 2). For two of our simulated

217 dispersers, no False Positive errors were identified. However, False Negative errors were 218 common.

219 Simulated disperser A (SD-A), artificially moved from its collection locality at Munglinup

220 SD-A was always identified as a resident when located in its collection locality, whether using

221 the highest or lowest PIC loci. We were able to identify SD-A to its collection locality when

222 using the three highest PIC loci available, whereas 11 loci were needed when using those having

223 the lowest PIC values. The minimum number of loci required to identify SD-A to its collection

224 locality $100 \%$ of the time did not vary between locations (Fig. 2).

\section{Simulated disperser B (SD-B), moved from Mallala}

226 SD-B was incorrectly identified as a disperser when located in its collection locality when using

227 the highest PIC loci but was always identified as a resident when using the lowest PIC loci.

228 When using the lowest PIC, 20 or more loci were needed to assign SD-B to its collection locality

$229100 \%$ of the time, while 27 loci were needed when using the highest PIC loci. SD-B was

230 identified as a disperser after using the 18 highest PIC loci; however, detection broke down

231 between 21 to 24 loci. When using the lowest PIC loci SD-B was not correctly assigned $1.66 \%$

232 of the time, and only when located in Orange. SD-B was not correctly assigned $14.94 \%$ of the

233 time when using the highest PIC loci (Fig. 2).

234 Simulated disperser C (SD-C), moved from Orange

235 SD-C was always identified as a resident when located in its collection locality whether using the

236 highest or lowest PIC loci. Fifteen loci were required to identify SD-C to its collection locality

$237100 \%$ of the time, whether using the highest or lowest PIC loci. When using the highest PIC loci, 
23821 out of 27 treatments correctly identified SD-C with 10 or more loci. For treatments from 1 to

23915 loci where SD-C was incorrectly identified, the location of SD-A contributed to the incorrect

240 identification. SD-C was not correctly assigned to Mallala $2.81 \%$ of the time when using the

241 highest PIC loci and was not correctly assigned only when located in Munglinup (Fig. 2).

\section{Literature search}

243 The studies we evaluated were published between 2005 and 2016. Studies that implemented

244 first-generation migrant detection in GeneClass2.0 to identify dispersers used on average $11.2 \pm$

2455.1 loci. For a subset of these data $(N=72)$, where global $F_{S T}$ was available, the average global

$246 F_{S T}$ was, $0.120 \pm 0.119$. There was no relationship between the global $F_{S T}$ of these studies and the

247 number of loci used when conducting genetic assignment tests (GLM, $\mathrm{t}_{71}=-1.312$; Fig. 3 ).

248 Figure 4 demonstrates that, despite the recent increase in population genetic papers based on

249 next-generation sequencing data, microsatellite data sets continue to be used for contemporary

250 disperser identification at a similar rate over the past eight years (more than 100 papers per year

251 during this period).

\section{Discussion}

253 Many studies have emphasised the need to have sufficient numbers of highly variable loci in

254 order to carry out population assignment tests with a high degree of confidence (Manel et al.

255 2002; Berry et al. 2004; Evanno et al. 2005). Here we artificially force dispersal between a set of

256 populations and test the power of the marker system (i.e. number of loci and levels of variability)

257 to identify dispersers. Our results show that we should be validating genetic assignment tests by

258 testing the power of the marker systems we use. Without validation of genetic data sets we

259 cannot be confident in the inferences we make about dispersal. Our results confirm that the 
260 number of loci is one of the best predictors that a marker system has adequate power to detect

261 real dispersal events (Smouse \& Chevillon 1998; Bernatchez \& Duchesne 2000). However, both

262 false positive (identified as a disperser when they are not one) and false negative (not identified

263 as a disperser when they are one) assignments can result even when using many loci with high

264 levels of polymorphism (e.g. simulated disperser B). The approach we present highlights the

265 number of false positive and false negative assignments (see Fig. 2).

266 Similar to previous studies (Bernatchez \& Duchesne 2000; Berry et al. 2004), our data indicated

267 that the number of loci used to identify dispersers strongly influences the results of contemporary

268 genetic disperser analysis. As more loci are added to an analysis the total information available

269 for discriminating between the genotype of an individual and the background population

270 increases, which increases the detectability of dispersers. Others have suggested that

271 approximately 10 loci are required to correctly identify dispersers when using assignment tests

272 with microsatellite data (Cornuet et al. 1999; Bernatchez \& Duchesne 2000); however, for our

273 study system, ideally 27 loci were required (Fig. 1). In our study, false negatives (i.e. not

274 identifying an individual as a migrant when they were) were more common than false positives

275 (i.e. when an individual was identified as a migrant when they were not). This difference

276 highlights the fact that every study requires an individual validation of the marker system in

277 order to be confident of identifying dispersers using genetic assignment tests.

278 Not surprisingly, when using loci with low PIC more loci are usually required to reach the same

279 level of discrimination than loci with high PIC because low PIC loci provide less discriminatory

280 power for the analysis. However, this is not always true as seen with individual B when using 21

281 to 24 of the highest PIC loci. A single locus can result in inconsistent assignment of dispersers

282 even when using a large number of loci. It is possible that a single locus can play a 
283 comparatively large role in distinguishing the multi-locus genotype of an individual. For

284 example, if an individual has alleles at a locus that are rare in its population but more common in

285 another population, inclusion of this locus may increase the incorrect assignment of that

286 individual. Post-hoc analyses, where locus 21 was removed, resulted in correct assignment of

287 SD-B for all locus combinations. This demonstrates how our approach can help to identify

288 problematic loci (i.e. those that yield inaccurate assignments). Although spurious results might

289 occur with any combination of loci, it is reasonable to assume that such results would generally

290 become less likely as more discriminatory information becomes available with an increased

291 number of loci.

292 Our results confirm those of previous studies (Cornuet et al. 1999; Berry et al. 2004) by showing

293 that it was easier to identify a disperser when they were moved into a population that had a

294 relatively high pairwise differentiation (e.g. I) from their collection locality. Although Berry et

295 al. (2004) clearly showed this effect in a natural system over a decade ago, there has been

296 surprisingly little response to these findings. If researchers were to take into account the

297 relationship between population differentiation (e.g. I, $F_{S T}$ ) and the number of loci required and

298 the ability to correctly identify dispersers, studies that use genetic assignment tests should use

299 more loci when their global $F_{S T}$ is lower. As shown by our analysis of the literature (Fig. 3),

300 studies with lower $F_{S T}$ values are not consistently using more loci to compensate for detection

301 ability. By not dealing with this relationship, published studies may have incorrectly identified

302 dispersers, potentially leading to misleading conclusions. To be confident of research outcomes,

303 we recommend that studies only using genetic assignment test to identify dispersers should

304 employ an approach similar to the Simulated Disperser Analysis that we describe here to validate

305 their marker panel. 
306 For a quick and stringent method capable of determining the number of loci necessary to

307 accurately identify dispersers (e.g. an approach that is able to distinguish individuals that are

308 easy to identify and hard to identify, such as SD-A and SD-B respectively) a 'worst-case

309 scenario' is necessary. This study showed that a worst-case scenario can be expected to occur

310 when individuals are moved to the most genetically similar population and the lowest PIC loci

311 are used. Therefore, for future studies to validate their disperser analysis, they could move

312 simulated dispersers to the most genetically similar population, start with the lowest PIC locus

313 and continue by adding the next lowest PIC locus (Fig. 5). Such a simulated dispersal scenario

314 makes biological sense given that genetic populations that have the lowest pairwise $F_{S T}$ values

315 are likely to be geographically closest to one another, and dispersers are more likely to move to a

316 population closer to their own rather than further away (Sutherland et al. 2000). If spurious

317 results are observed (false positive), such as when using the $21^{\text {st }}$ locus to detect SD-B caused

318 consistently incorrect results in our study, the offending loci may require greater scrutiny

319 because this could result from mis-scoring.

320 In studies where some information on levels of genetic differentiation and levels of loci

321 polymorphism are known, a preliminary "true" simulation study (i.e. based on synthetic samples)

322 can potentially provide guidance on sample sizes needed to correctly identify dispersers.

323 However, a simulated dispersal approach is applicable in cases where there is no prior

324 information on levels of genetic differentiation and levels of loci polymorphism. It is also

325 important to note that a simulated dispersal approach will only be valuable when estimates of the 326 underlying allele frequencies are gained from a robust sampling protocol. 


\section{Conclusion}

328 An increase in the number of loci and the pairwise differentiation (e.g. I, $F_{S T}$ ) between a

329 disperser's collection locality and its final destination increases the accuracy of genetically

330 assigning the disperser (Cornuet et al. 1999; Bernatchez \& Duchesne 2000; Berry et al. 2004).

331 Our study showed similar patterns to previous research on natural systems (Berry et al. 2004),

332 however, the magnitude of the effect varied. Although basic patterns may be discernible when

333 determining the number of loci necessary to accurately identify dispersers, the unique nature of

334 natural systems means that every study system will be different. By validating our genetic

335 disperser analysis, we were able to determine the number of loci required to increase the

336 accuracy of identifying dispersers in a wild population without the need for direct measures of

337 dispersal (e.g. tracking data, etc). Although next-generation sequencing data sets are becoming

338 more prevalent in population genetic studies, we demonstrate here that microsatellite data are

339 still commonly used to estimate contemporary dispersal and believe they will continue to do so

340 (De Barba et al 2016). Although our demonstration of this approach used microsatellite data, the

341 process is also applicable analyses of next-generation sequencing data sets.

\section{Acknowledgments}

343 We thank the Ramaciotti Centre for Gene Function Analysis for 454 sequencing and also Simon

344 Griffith for the use of his laboratory space. Thanks to Andrew Woolnough, Ron Sinclair, John

345 Tracey and the WA Department of Agriculture and Food, for help collecting samples. We would

346 like to acknowledge the starlings that were killed as part of a control program and used in this

347 research. 


\section{References}

349 Abdelkrim J, Robertson BC, Stanton J-AL, Gemmell NJ (2009) Fast, cost-effective development

350 of species-specific microsatellite markers by genomic sequencing. BioTechniques, 46, 185$351 \quad 192$.

352 Bates DM, Maechler M, Bolker B (2012) lme4: Linear mixed-effects models using S4 classes.

353 Bernatchez L, Duchesne P (2000) Individual-based genotype analysis in studies of parentage and 354 population assignment: how many loci, how many alleles? Canadian Journal of Fisheries 355 and Aquatic Sciences, 57, 1-12.

356 Berry O, Tocher MD, Sarre SD (2004) Can assignment tests measure dispersal? Molecular $357 \quad$ Ecology, 13, 551-561.

358 Bohonak AJ (1999) Dispersal, gene flow, and population structure. The Quarterly Review of $359 \quad$ Biology, 74, 21-45.

360 Broquet T, Petit EJ (2009) Molecular Estimation of Dispersal for Ecology and Population 361 Genetics. Annual Review of Ecology, Evolution, and Systematics, 40, 193-216.

362 Cagnacci F, Boitani L, Powell RA, Boyce MS (2010) Animal ecology meets GPS-based

363 radiotelemetry: a perfect storm of opportunities and challenges. Philosophical transactions 364 of the Royal Society B: Biological sciences, 365, 2157-62.

365 Clobert J, Baguette M, Benton TG, Bullock JM (2012) Dispersal Ecology and Evolution. Oxford $366 \quad$ University Press.

367 Cornuet JM, Piry S, Luikart G, Estoup A, Solignac M (1999) New methods employing 368 multilocus genotypes to select or exclude populations as origins of individuals. Genetics, $369 \quad \mathbf{1 5 3}, 1989-2000$. 
370 De Barba M, Miquel C, Lobréaux S, Quenette PY, Swenson JE, Taberlet P (2016) High-

371 throughput microsatellite genotyping in ecology: improved accuracy, efficiency,

372 standardization and success with low-quantity and degraded DNA. Molecular Ecology

$373 \quad$ Resources, 17(3), 492-507

374 Dewar RC, Sherwin WB, Thomas E, Holleley CE, Nichols RA (2011) Predictions of single-

375 nucleotide polymorphism differentiation between two populations in terms of mutual

376 information. Molecular ecology, 20, 3156-66.

377 Dzialak MR, Lacki MJ, Larkin JL, Carter KM, Vorisek S (2005) Corridors affect dispersal

378 initiation in reintroduced peregrine falcons. Animal Conservation, 8, 421-430.

379 Evanno G, Regnaut S, Goudet J (2005) Detecting the number of clusters of individuals using the $380 \quad$ software STRUCTURE: a simulation study. Molecular ecology, 14, 2611-20.

381 Excoffier L, Laval G, Schneider S (2005) Arlequin (version 3.0): an integrated software package

382 for population genetics data analysis. Evolutionary Bioinformatics Online, 1, 47-50.

383 Fahrig L, Merriam G (1994) Conservation of Fragmented Populations. Conservation Biology, 8, $384 \quad 50-59$.

385 Gortat T, Panagiotopoulou H, Gryczyńska-Siemiątkowska A et al. (2009) The spatial genetic 386 structure of bank vole (Myodes glareolus) and yellow-necked mouse (Apodemus flavicollis) 387 populations: The effect of distance and habitat barriers. Animal Biology, 59, 169-187.

388 Goudet J, Perrin N, Waser P (2002) Tests for sex-biased dispersal using bi-parentally inherited 389 genetic markers. Molecular Ecology, 11, 1103-1114.

390 Harris S, Cresswell WJ, Forde PG et al. (1990) Home-range analysis using radio-tracking data-a 391 review of problems and techniques particularly as applied to the study of mammals. 
393 Jombart T, Devillard S, Dufour A-B, Pontier D (2008) Revealing cryptic spatial patterns in 394 genetic variability by a new multivariate method. Heredity, 101, 92-103.

395 Keller D, Brodbeck S, Flöss I, Vonwil G, Holderegger R (2010) Ecological and genetic 396 measurements of dispersal in a threatened dragonfly. Biological Conservation, 143, 26583972663.

398 Manel S, Berthier P, Luikart G (2002) Detecting Wildlife Poaching: Identifying the Origin of 399 Individuals with Bayesian Assignment Tests and Multilocus Genotypes. Conservation $400 \quad$ Biology, 16, 650-659.

Meglécz E, Costedoat C, Dubut V et al. (2010) QDD: a user-friendly program to select 402 microsatellite markers and design primers from large sequencing projects. Bioinformatics 403 (Oxford, England), 26, 403-4.

Nagy S, Poczai P, Cernák I et al. (2012) PICcalc: an online program to calculate polymorphic 405 information content for molecular genetic studies. Biochemical Genetics, 50, 670-2.

Nakagawa S, Schielzeth H (2013) A general and simple method for obtaining $\mathrm{R}^{2}$ from 407 generalized linear mixed-effects models. Methods in Ecology and Evolution, 4, 133-142

Neilan BA, Wilton AN, Jacobs D (1997) A universal procedure for primer labelling of 409 amplicons. Nucleic Acids Research, 25, 2938-2939.

410 Paetkau D, Slade R, Burden M, Estoup A (2004) Genetic assignment methods for the direct, 411 real-time estimation of migration rate: a simulation-based exploration of accuracy and 412 power. Molecular Ecology, 13, 55-65.

413 Peakall R, Smouse PE (2006) genalex 6: genetic analysis in Excel. Population genetic software 
414 for teaching and research. Molecular Ecology Notes, 6, 288-295.

415 Piry S, Alapetite A, Cornuet J-M et al. (2004) GENECLASS2: a software for genetic assignment 416 and first-generation migrant detection. The Journal of heredity, 95, 536-9.

417 Porras-Hurtado L, Ruiz Y, Santos C et al. (2013) An overview of STRUCTURE: applications, 418 parameter settings, and supporting software. Frontiers in Genetics, 4, 98.

419 Pritchard JK, Stephens M, Donnelly P (2000) Inference of Population Structure Using $420 \quad$ Multilocus Genotype Data. Genetics, 155, 945-959.

421 Pruett-Jones SG, Lewis MJ (1990) Sex ratio and habitat limitation promote delayed dispersal in 422 superb fairy-wrens. Nature, 348, 541-542.

423 R Development Core Team (2012) R: A language and environment for statistical computing. 424 Vienna, Austria. URL https://www.R-project.org/.

425 Rannala B, Mountain JL (1997) Detecting immigration by using multilocus genotypes.

426 Proceedings of the National Academy of Sciences of the United States of America, 94, 9197 $427-9201$.

428 Rollins LA, Woolnough AP, Wilton AN, Sinclair R, Sherwin WB (2009) Invasive species can’t 429 cover their tracks: using microsatellites to assist management of starling (Sturnus vulgaris) 430 populations in Western Australia. Molecular Ecology, 18, 1560-1573.

431 Rousset F (2008) genepop'007: a complete re-implementation of the genepop software for 432 Windows and Linux. Molecular Ecology Resources, 8, 103-6.

433 Rozen S, Skaletsky H (2000) Primer3 on the WWW for general users and for biologist 434 programmers. In: Bioinformatics methods and protocols: methods in molecular biology.

435 (eds Misener S, Krawetz SA), pp. 365-386. Humana Press, Totowa, NJ. 
436 Sandercock BK (2003) Estimation of survival rates for wader populations: a review of mark437 recapture methods. Wader Study Group Bullitin2, 100, 163-174.

438 Schwartz MK, McKelvey KS (2008) Why sampling scheme matters: the effect of sampling 439 scheme on landscape genetic results. Conservation Genetics, 10, 441-452.

440 Selonen V, Hanski IK, Painter JN (2010) Gene flow and natal dispersal in the Siberian flying 441 squirrel based on direct and indirect data. Conservation Genetics, 11, 1257-1264.

442 Sherwin WB, Jabot F, Rush R, Rossetto M (2006) Measurement of biological information with 443 applications from genes to landscapes. Molecular ecology, 15, 2857-69.

444 Sherwin WB, Chao A, Jost L, Smouse PE (2017) Information Theory Broadens the Spectrum of 445 Molecular Ecology and Evolution. Trends in Ecology and Evolution (Accepted 27 Oct 446 2017, TREE-D-17-00068).

447 Smouse PE, Chevillon C (1998) Analytical aspects of population-specific DNA fingerprinting 448 for individuals. Journal of Heredity, 89, 143-150.

Sutherland GD, Harestad AS, Price K, Lertzman KP (2000) Scaling of Natal Dispersal Distances 450 in Terrestrial Birds and Mammals. Conservation Ecology, 4, 1-36.

Walsh J, Kovach AI, Babbitt KJ, O’Brien KM (2012) Fine-scale population structure and asymmetrical dispersal in an obligate salt-marsh passerine, the Saltmarsh Sparrow (Ammodramus caudacutus). The Auk, 129, 247-258.

Waser, PM, JD Hadfield (2011) How Much Can Parentage Analyses Tell Us about Precapture Dispersal?. Molecular Ecology, 20, 1277-88. https://doi.org/10.1111/j.1365294X.2011.05002.x.

457 Waser, PM, D Paetkau, C Strobeck (2001) Estimating interpopulation dispersal rates. Pp 484- 
458497 in JL Gittleman, SM Funk, DW Macdonald and RK Wayne, eds. Carnivore 459 Conservation. Cambridge University Press)

460 Whitlock MC, McCauley DE (1999) Indirect measures of gene flow and migration: FST not 461 equal to $1 /(4 \mathrm{Nm}+1)$. Heredity, $\mathbf{8 2}, 117-125$.

462 Wickham H (2009) ggplot2: elegant graphics for data analysis. Springer New York.

463 Zuur AF, Ieno EN, Walker NJ, Saveliev AA, Smith GM (2009) Mixed effects models and

464 extensions in ecology with R (M Gail, K Krickeberg, JM Samet, A Tsiatis, W Wong, Eds,).

465 Springer, United Kingdom.

466 


\section{Figure 1}

Probability of correctly identifying a simulated disperser by the number of loci used.

Genetic assignment tests were used to determine whether three common starlings (Sturnus vulgaris) from three genetically distinct collection localities would be identified as dispersers when their movement to a new location was simulated. Shown is the relationship between the probabilities (y-axis), from a Generalised Linear Mixed Model, of correctly identifying a simulated disperser plotted against an increase in the number of loci used. The top line indicates the response when using loci with the highest Polymorphic Information Content (PIC) first (blue line), and the bottom line indicates the response when using loci with the lowest PIC first (red line). The coloured shading corresponding to each line encompasses the upper and lower confidence intervals of the models.

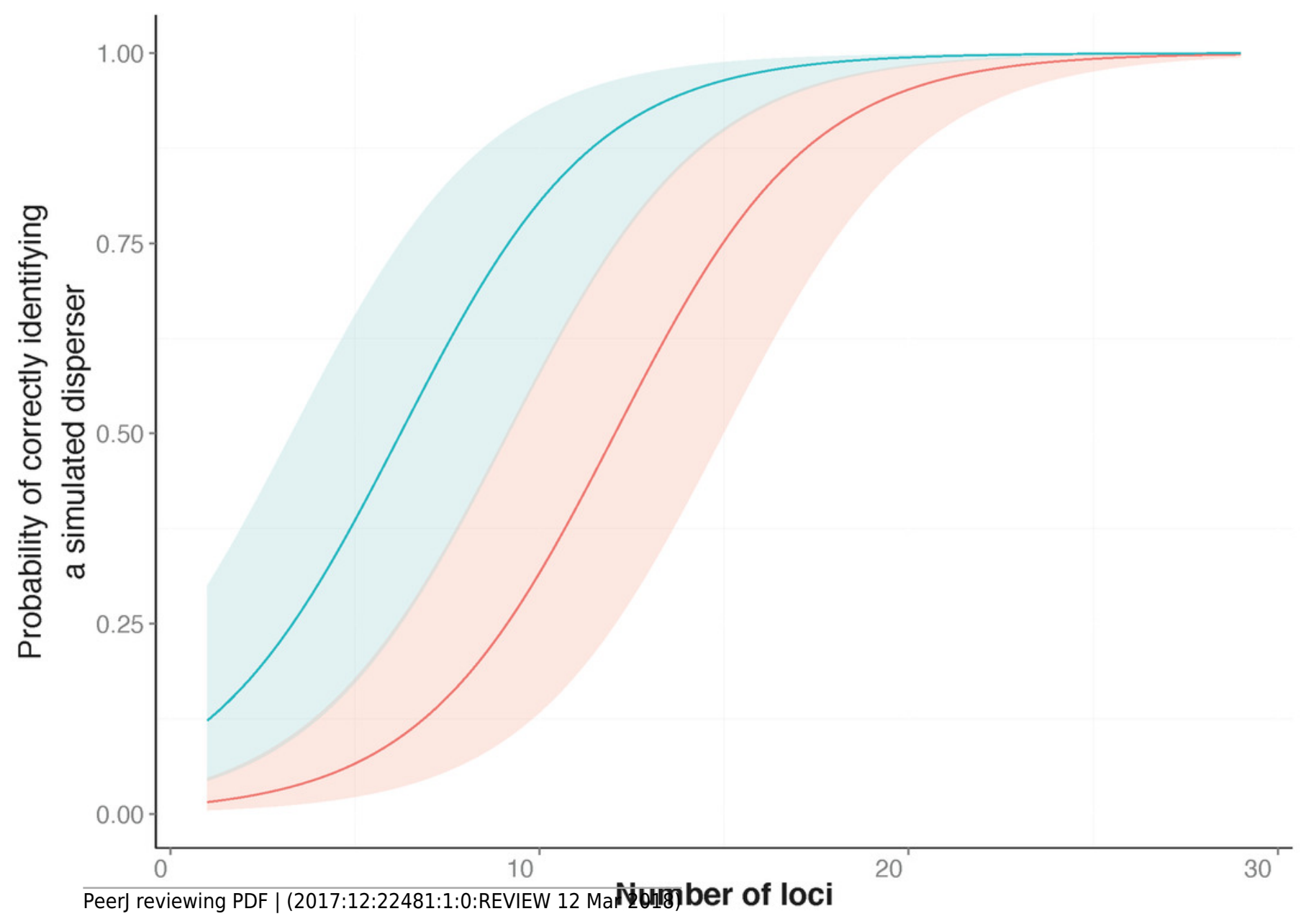




\section{Figure 2}

Heatmap showing the assignment status of each simulated disperser.

Genetic assignment tests were used to determine whether three common starlings (Sturnus vulgaris) from three genetically distinct collection localities $(A=$ Munglinup, $B=$ Mallala, $C=$ Orange) would be identified as dispersers when their movement to a new location was simulated. Each small coloured rectangle represents the results of a genetic assignment test; blue, individual was correctly assigned to its collection locality; red, individual was not recognised as a disperser or resident; orange, individual was identified as a disperser but assigned to the incorrect collection locality. The three panels represent the results for simulated disperser A, C and B. Within each panel the top heat map shows the results when first using loci with the highest Polymorphic Information Content (PIC) and the bottom heat map shows the results when first using loci with the lowest PIC. The grey arrows below each inset show the direction the loci were sorted, from high PIC to low PIC (top) or vice versa (bottom). Each inset is sorted on the $y$-axis by the location the individual was found and the movement treatment. Each row corresponds to a different treatment. The treatment order of each simulated migrant's heat map row can be found in Supplementary Table 4. 
Assignment of simulated disperser $\mathrm{A}$

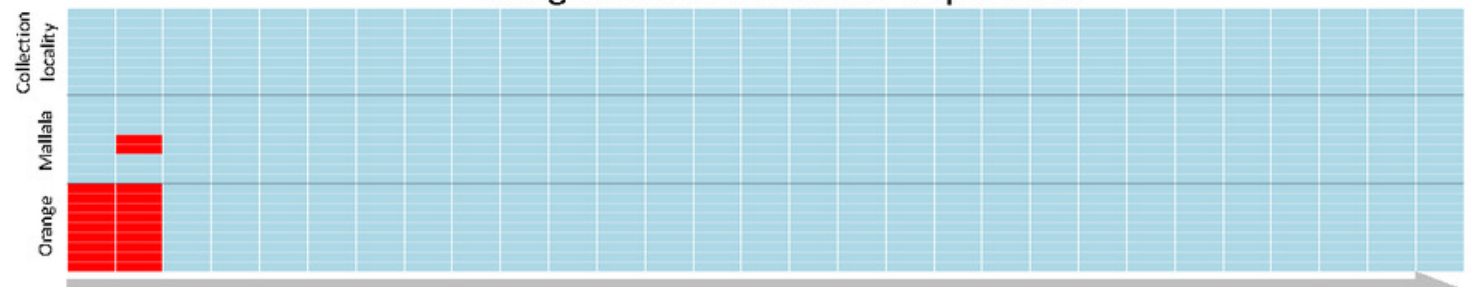

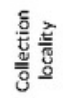

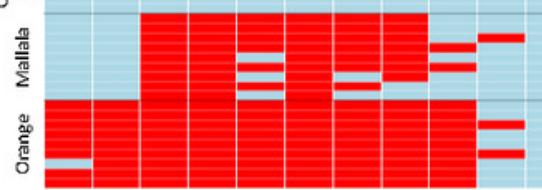

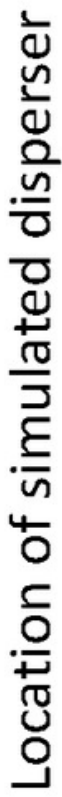

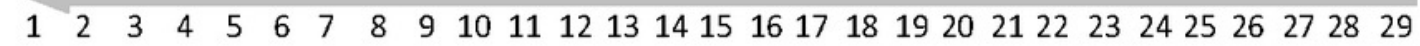
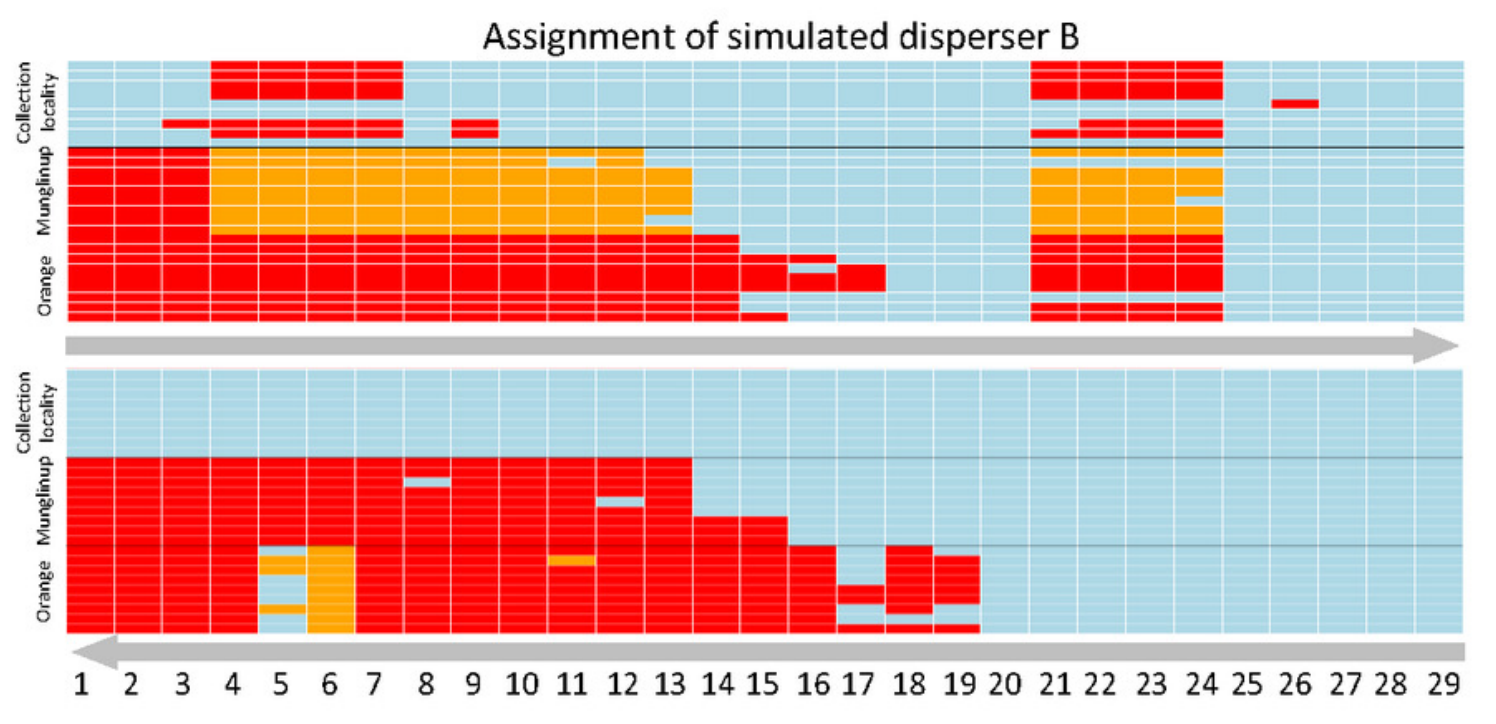
Assignment of simulated disperser C
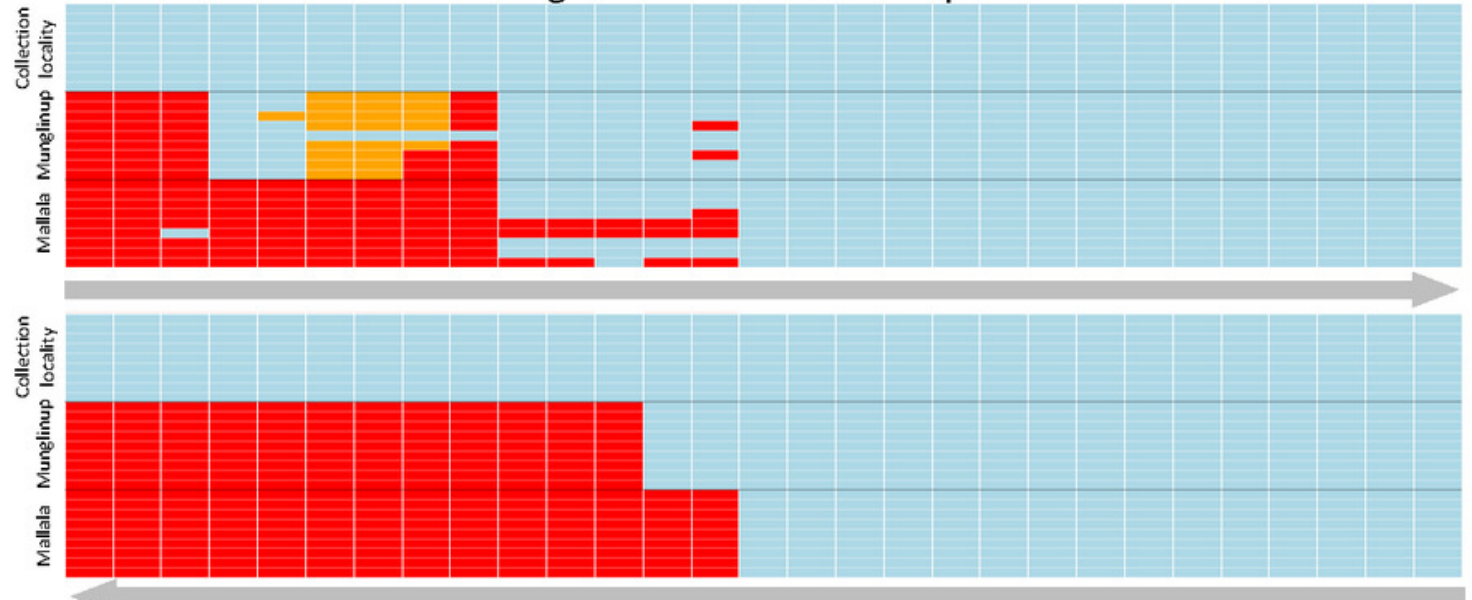

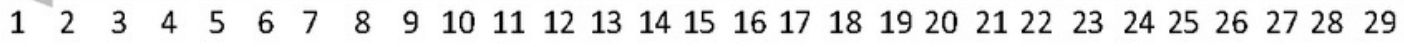
Number of loci 
Figure 3

Data extracted during a literature review showing the relationship between the number of loci used and $F_{S T}$ value.

A literature review was conducted to gather data on studies that used genetic assignment tests in GeneClass2.0 and their global $F_{S T}$. This graph shows the non-significant relationship $\left(G L M, t_{71}=-1.312\right)$ between the number of loci used and global $F_{S T}$ of 72 datasets. The grey band shows the $95 \%$ confidence interval on the fitted values.

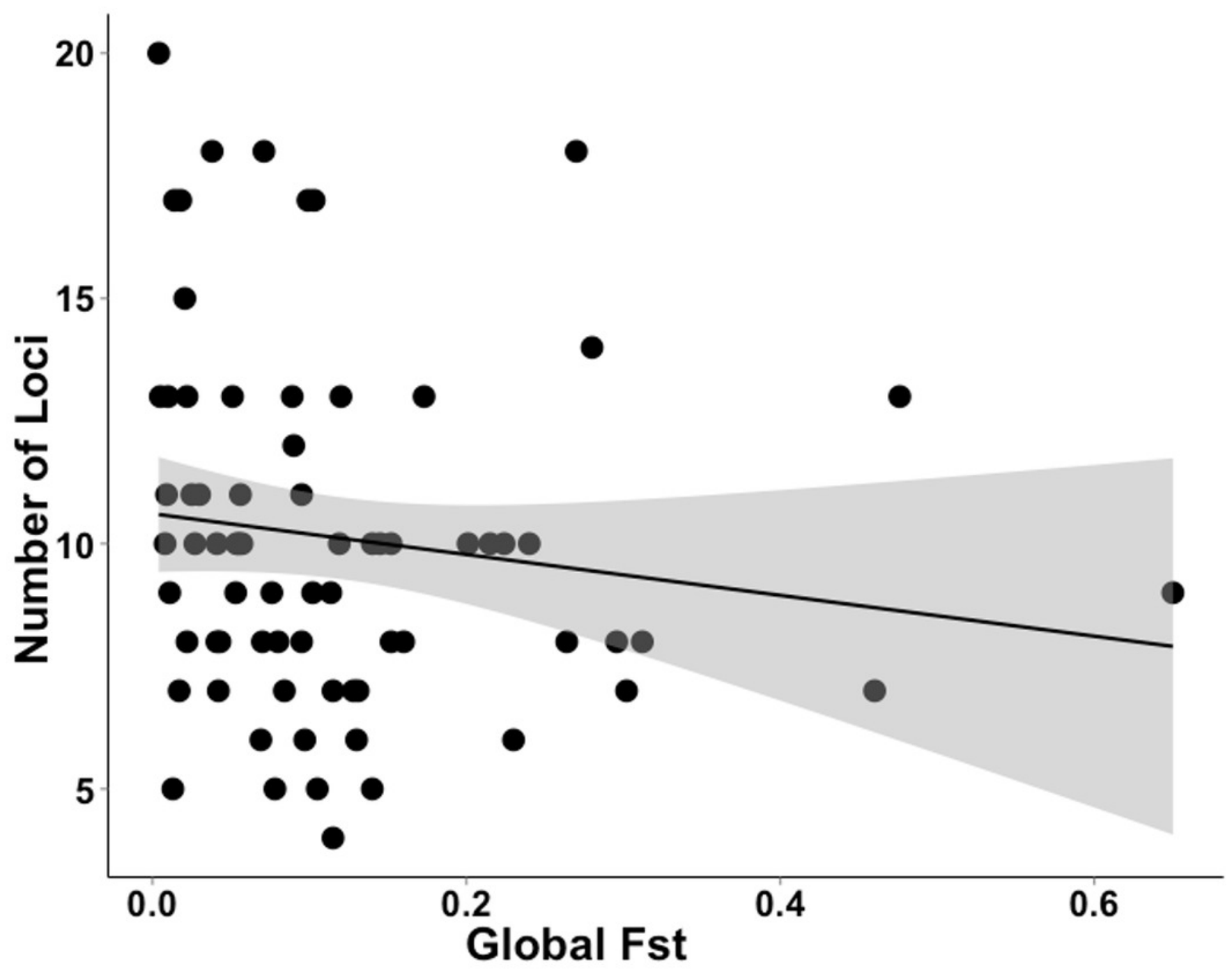


Figure 4

Number of papers that cited Piry et al. 2016 and contained the search term 'microsatellite' each year between 2005 and 2016.

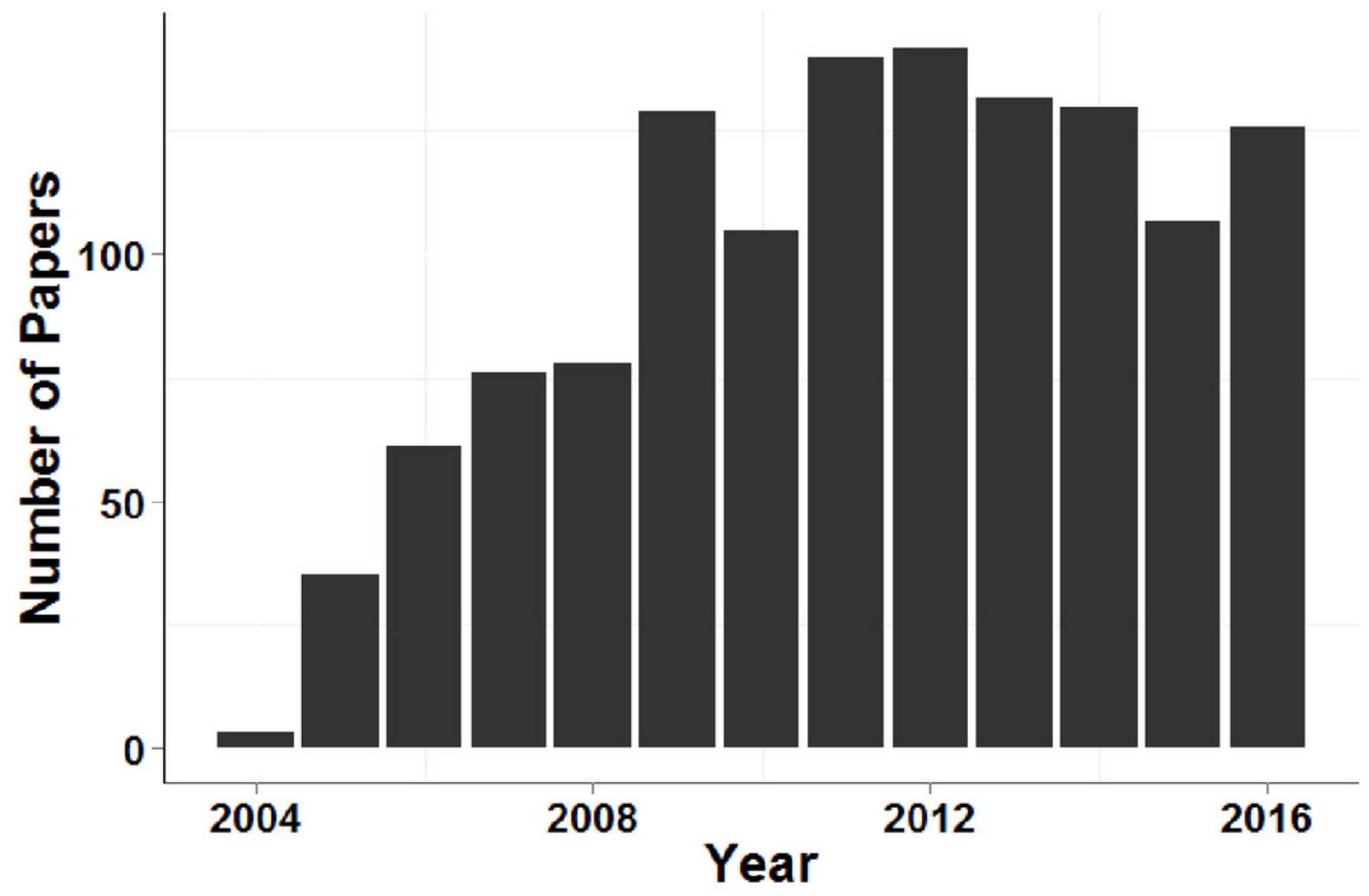


Figure 5

Recommended workflow indicating the steps necessary for validating genetic assignment tests. 
1) Sort loci by PIC (or equivalent measure) and order.

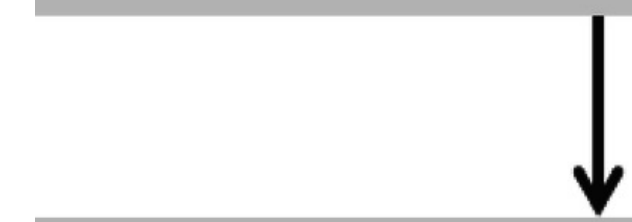

2) Assign simulated disperser for each population.

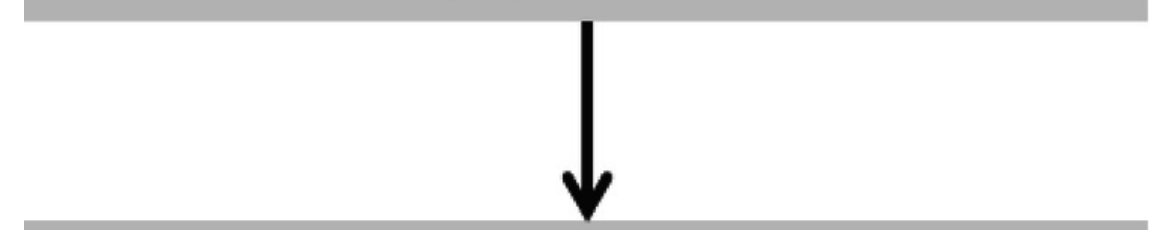

3) Move each simulated disperser to the most genetically related population (lowest pairwise Fst).

4) Run genetic assignment test.

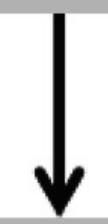

5) Remove best locus.

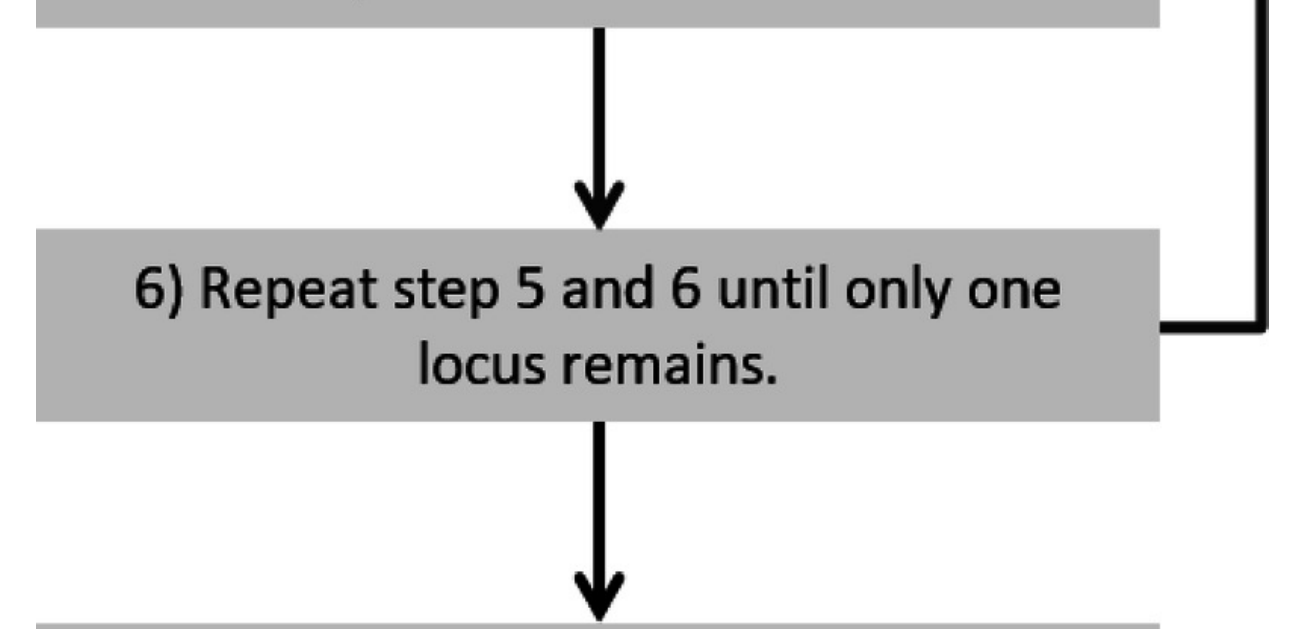

7) Visualise correct and incorrect

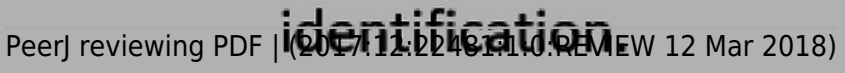




\section{Table $\mathbf{1}$ (on next page)}

List of simulated disperser movement combination used in analysis.

Simulated dispersers were moved in every possible combination relative to other simulated dispersers creating a total of $27\left(3^{3}\right)$ treatments. This table indicates the location of each simulated disperser for each of the 27 treatments. Treatment 0 is the collection locality of each of the simulated dispersers before any simulated movement has taken place. For example, for treatment 0 , simulated disperser $A$ is located in Munglinup, $B$ is located in Mallala and $C$ in Orange (their collection localities). In treatment 1 simulated disperser $A$ stayed in Munglinup while B and C were both moved from their collection localities (treatment 0 ) to Munglinup. 
Table 1. Simulated dispersers were moved in every possible combination relative to other simulated dispersers creating a total of $27\left(3^{3}\right)$ treatments. This table indicates the location of each simulated disperser for each of the 27 treatments. Treatment 0 is the collection locality of each of the simulated dispersers before any simulated movement has taken place. For example, for treatment 0 , simulated disperser $\mathrm{A}$ is located in Munglinup, B is located in Mallala and C in Orange (their collection localities). In treatment 1 simulated disperser A stayed in Munglinup while B and C were both moved from their collection localities (treatment 0) to Munglinup.

\begin{tabular}{llll}
\hline Treatment & Munglinup & Mallala & Orange \\
\hline $\mathbf{0}$ & $\mathrm{A}$ & $\mathrm{B}$ & $\mathrm{C}$ \\
$\mathbf{1}$ & $\mathrm{ABC}$ & 0 & 0 \\
$\mathbf{2}$ & 0 & $\mathrm{ABC}$ & 0 \\
$\mathbf{3}$ & 0 & 0 & $\mathrm{ABC}$ \\
$\mathbf{4}$ & $\mathrm{AB}$ & $\mathrm{C}$ & 0 \\
$\mathbf{5}$ & $\mathrm{AB}$ & 0 & $\mathrm{C}$ \\
$\mathbf{6}$ & $\mathrm{AC}$ & $\mathrm{B}$ & 0 \\
$\mathbf{7}$ & $\mathrm{AC}$ & 0 & $\mathrm{~B}$ \\
$\mathbf{8}$ & $\mathrm{BC}$ & $\mathrm{A}$ & 0 \\
$\mathbf{9}$ & $\mathrm{BC}$ & 0 & $\mathrm{~A}$ \\
$\mathbf{1 0}$ & 0 & $\mathrm{BC}$ & $\mathrm{A}$ \\
$\mathbf{1 1}$ & $\mathrm{A}$ & $\mathrm{BC}$ & 0 \\
$\mathbf{1 2}$ & $\mathrm{A}$ & 0 & $\mathrm{BC}$ \\
$\mathbf{1 3}$ & 0 & $\mathrm{~A}$ & $\mathrm{BC}$ \\
$\mathbf{1 4}$ & 0 & $\mathrm{AB}$ & $\mathrm{C}$ \\
$\mathbf{1 5}$ & $\mathrm{C}$ & $\mathrm{AB}$ & 0 \\
$\mathbf{1 6}$ & 0 & $\mathrm{AC}$ & $\mathrm{B}$ \\
$\mathbf{1 7}$ & $\mathrm{B}$ & $\mathrm{AC}$ & 0 \\
$\mathbf{1 8}$ & 0 & $\mathrm{C}$ & $\mathrm{AB}$ \\
$\mathbf{1 9}$ & $\mathrm{C}$ & 0 & $\mathrm{AB}$ \\
$\mathbf{2 0}$ & $\mathrm{B}$ & 0 & $\mathrm{AC}$ \\
$\mathbf{2 1}$ & 0 & $\mathrm{~B}$ & $\mathrm{AC}$ \\
$\mathbf{2 2}$ & $\mathrm{C}$ & $\mathrm{A}$ & $\mathrm{B}$ \\
$\mathbf{2 3}$ & $\mathrm{B}$ & $\mathrm{C}$ & $\mathrm{A}$ \\
$\mathbf{2 4}$ & $\mathrm{C}$ & $\mathrm{B}$ & $\mathrm{A}$ \\
$\mathbf{2 5}$ & $\mathrm{A}$ & $\mathrm{C}$ & $\mathrm{B}$ \\
$\mathbf{2 6}$ & $\mathrm{B}$ & $\mathrm{A}$ & $\mathrm{C}$ \\
\hline & & & \\
\hline
\end{tabular}




\section{Table 2 (on next page)}

Results of model testing the impact of variables on correctly identifying simulated dispersers.

Genetic assignment tests were used to determine whether three common starlings (Sturnus vulgaris) from three genetically distinct collection localities would be identified as dispersers when their movement to a new location was simulated. Given are results from a Generalised Linear Mixed Model used to determine the effect of the predictor variables, the number of loci, loci with high or low Polymorphic Information Content (PIC) and simulated disperser movement, on the ability to correctly identify the status of simulated dispersers. The relationship of the response variable (simulated disperser's status correctly identified by a genetic assignment test) was tested by specifying movement in two ways. The first model specified movement as the location to which a simulated disperser was moved (i.e. 'In Mallala' means a simulated disperser was moved from its collection locality to Mallala). Genetic distance (I) between the two locations (collection locality and simulated dispersal location) was used as a predictor variable in the second model. $C=$ coefficient; $S E=$ standard error; $Z$ = test statistic. Model fit was estimated from marginal $\left(R_{G L M M /(m)}^{2}\right)$ and conditional ( $R_{\text {GLMM/(C) }}^{2}$ ) coefficients of determination; $R_{\text {GLMM/(m) }}^{2}$ estimate model fit using fixed effects only, while $R_{\text {GLMM(c) }}^{2}$ estimates model fit including both fixed and random effects. 
Table 2. Genetic assignment tests were used to determine whether three common starlings (Sturnus vulgaris) from three genetically distinct collection localities would be identified as dispersers when their movement to a new location was simulated. Given are results from a Generalised Linear Mixed Model used to determine the effect of the predictor variables, the number of loci, loci with high or low Polymorphic Information Content (PIC) and simulated disperser movement, on the ability to correctly identify the status of simulated dispersers. The relationship of the response variable (simulated disperser's status correctly identified by a genetic assignment test) was tested by specifying movement in two ways. The first model specified movement as the location to which a simulated disperser was moved (i.e. 'In Mallala' means a simulated disperser was moved from its collection locality to Mallala). Genetic distance (I) between the two locations (collection locality and simulated dispersal location) was used as a predictor variable in the second model. $C=$ coefficient; $S E=$ standard error; $Z=$ test statistic. Model fit was estimated from marginal $\left(R_{\mathrm{GLMM} /(\mathrm{m})}\right)$ and conditional $\left(R_{\mathrm{GLMM} /(\mathrm{c})}^{2}\right)$ coefficients of determination; $R_{\mathrm{GLMM} /(\mathrm{m})}^{2}$ estimate model fit using fixed effects only, while $R_{\text {GLMM/(c) }}^{2}$ estimates model fit including both fixed and random effects.

\begin{tabular}{lllll}
\hline Response Variable & Predictor Variable & $\boldsymbol{C}$ & $\boldsymbol{S E}$ & $\boldsymbol{Z}$ \\
\hline $\begin{array}{l}\text { Simulated disperser } \\
\text { status correctly identified } \\
\text { by genetic assignment } \\
\text { test }\end{array}$ & Number of loci & 0.30 & 0.01 & 26.98 \\
& & & & \\
$\left(\boldsymbol{R}_{\mathbf{G L M M} /(\mathbf{m})}^{2}=\mathbf{0 . 6 2 6 0} ;\right.$ & Highest / lowest PIC loci used & 1.32 & 0.11 & 11.62 \\
$\boldsymbol{R}_{\text {GLMM/(c) }}^{2} \mathbf{0 . 8 0 3 5 )}$ & Moved to Mallala & & & \\
& Moved to Munglinup & -4.75 & 0.24 & -19.91 \\
& Moved to Orange & -3.18 & 0.20 & -15.71 \\
& & -5.75 & 0.25 & -23.31 \\
\hline
\end{tabular}


Simulated disperser

Number of loci

$\begin{array}{lll}0.38 & 0.01 & 25.39\end{array}$

status correctly identified

by genetic assignment

test

$\left(R_{\mathrm{GLMM} /(\mathrm{m})}^{2}=0.7390 ;\right.$

Highest / lowest PIC loci used

2.18

$0.14 \quad 15.07$

$\left.R^{2}{ }_{\mathrm{GLMM} /(\mathrm{c})}=0.8039\right)$

$37.16 \quad 3.03$

12.25 